\title{
Imaging Macrophage Accumulation in a Murine Model of Chronic Pancreatitis with ${ }^{125}$ I-Iodo-DPA-713 SPECT/CT
}

\author{
Catherine A. Foss ${ }^{1}$, Liansheng Liu $^{2}$, Ronnie C. Mease ${ }^{1}$, Haofan Wang ${ }^{1}$, Pankaj Pasricha ${ }^{2}$, and Martin G. Pomper ${ }^{1}$ \\ ${ }^{I}$ Russell H. Morgan Department of Radiology and Radiological Science, Johns Hopkins Medical Institutions, Baltimore, Maryland; \\ and ${ }^{2}$ Center for Neurogastroenterology, Department of Medicine, Johns Hopkins Medical Institutions, Baltimore, Maryland
}

\begin{abstract}
Pancreatitis remains a diagnostic challenge in patients with mild to moderate disease, with current imaging modalities being inadequate. Given the prominent macrophage infiltration in chronic pancreatitis, we hypothesized that ${ }^{125}$ /-iodo-DPA-713, a small-molecule radiotracer that specifically targets macrophages, could be used with SPECT/CT to image pancreatic inflammation in a relevant experimental model. Methods: Chronic pancreatitis was induced with cerulein in C57BL/6 mice, which were contrasted with saline-injected control mice. The animals were imaged at $7 \mathrm{wk}$ after induction using $\mathrm{N}, \mathrm{N}$-diethyl-2-(2(3-125/-iodo-4-methoxyphenyl)-5,7-dimethylpyrazolo[1,5-a]pyrimidin3-yl)acetamide ( ${ }^{125}$-iodo-DPA-713) SPECT/CT or ${ }^{18} \mathrm{~F}-\mathrm{FDG}$ PET/CT. The biodistribution of ${ }^{125}$ /-iodo-DPA-713 was determined under the same conditions, and a pair of mice was imaged using a fluorescent analog of 125 I-iodo-DPA-713, DPA-713-IRDye800CW, for correlative histology. Results: Pancreatic ${ }^{125}$-iodo-DPA-713 uptake was significantly higher in treated mice than control mice $(5.17 \% \pm 1.18 \%$ vs. $2.41 \% \pm 0.34 \%$ injected dose/g, $P=0.02$ ), as corroborated by imaging. Mice imaged with ${ }^{18}$ F-FDG PET/CT showed cerulein-enhanced pancreatic uptake in addition to a moderate signal from healthy pancreas. Near-infrared fluorescence imaging with DPA-713IRDye800CW showed strong pancreatic uptake, focal liver uptake, and gastrointestinal uptake in the treated mice, whereas the control mice showed only urinary excretion. Ex vivo fluorescence microscopy revealed a large influx of macrophages in the pancreas colocalizing with the retained fluorescent probe in the treated but not the control mice. Conclusion: These data support the application of both ${ }^{125}$ I-iodo-DPA-713 SPECT/CT and DPA-713-IRDye800CW near-infrared fluorescence to delineate pancreatic, liver, or intestinal inflammation in living mice.
\end{abstract}

Key Words: macrophages; DPA-713; pancreatitis; SPECT-CT

J Nucl Med 2017; 58:1685-1690

DOI: 10.2967/jnumed.117.189571

\section{A}

cute pancreatitis occurs at a rate of $13-45 / 100,000$ persons (1), and approximately 50/100,000 individuals develop chronic disease (2). Mortality from chronic pancreatitis increases with its duration, with survival at 5, 10, and 20 y being 97\%, 70\%$86.3 \%$, and $45 \%-63 \%$, respectively (3). The overall cost of care in 2012 was $\$ 150$ million in the United States (4). Chronic pancreatitis is relatively easy to define with a combination of pathologic

Received Jan. 6, 2017; revision accepted May 1, 2017.

For correspondence or reprints contact: Catherine A. Foss, Johns Hopkins Medical Institutions, 1550 Orleans St., 493 CRB II, Baltimore, MD 21287.

E-mail: cfoss1@jhmi.edu

Published online May 18, 2017.

COPYRIGHT (C 2017 by the Society of Nuclear Medicine and Molecular Imaging. and functional findings. However, given the location and nature of the organ, it is difficult to obtain a biopsy of the pancreas in a safe and convenient manner. Although diagnosis by conventional radiologic imaging (CT or MRI) is straightforward in florid cases, it is challenging in patients with subtler disease because of the relatively nonspecific nature of the clinical features and incomplete validation of imaging findings (5). In addition, there is also a need for improved imaging to monitor active inflammation for response to treatment or for objective correlation with flare-ups of pain.

Although inflammation is universal in pancreatitis regardless of etiology and is a biomarker for disease severity (6-9), clinical imaging for management of pancreatitis is primarily undertaken with anatomic techniques such as CT, ultrasound, and endoscopy, which can detect only gross correlates (fluid or phlegmon) of the inflammatory process $(10-14)$. PET with ${ }^{18} \mathrm{~F}-\mathrm{FDG}$ is a sensitive molecular imaging technique that has been tested in chronic pancreatitis, but this modality detects all glycolytic metabolism, including that from the unaffected pancreas itself $(15,16)$. Macrophage activity is an attractive and novel target for biomarker development since macrophages represent approximately one third of the inflammatory infiltrate in chronic pancreatitis (17).

The translocator protein (TSPO) found on the mitochondrial membrane and in all steroidogenic tissues, including the heart and liver (18-20), is upregulated in activated monocytes and macrophages (21). TSPO has been targeted to image neuroinflammation, cancer, and inflammation associated with coronary artery disease (19,22-31). Here, we used N,N-diethyl-2-(2-(3-125I-iodo-4-methoxyphenyl)-5,7-dimethylpyrazolo[1,5-a]pyrimidin-3-yl)acetamide ( ${ }^{125}$ I-iodo-DPA-713), a second-generation TSPO radioligand that we previously developed $(30,31)$, to target macrophages associated with pancreatitis and demonstrate proof of the concept that it can be used as an imaging tool in a mouse model of chronic pancreatitis. We also showed that the mechanism by which ${ }^{125}$ I-iodo-DPA-713 binds to the inflamed pancreas is related to TSPO-independent trapping within macrophages.

\section{MATERIALS AND METHODS}

\section{Induction of Chronic Pancreatitis}

Animal procedures were performed in accordance with the regulations of the Johns Hopkins Animal Care and Use Committee under an approved protocol. Twenty male C57BL/6B mice (6 wk old) were obtained from Jackson Laboratories. The mice were housed in a specific pathogen-free facility under controlled conditions of temperature and humidity with a 12/12 light/dark cycle and were maintained on standard laboratory chow (Teklad 8604; Harlan Laboratories) with free access to water. They were randomly assigned to either of 2 groups: a chronic pancreatitis group or a control group. Chronic pancreatitis was induced by intraperitoneal injection of cerulein (Sigma) at a dose of $50 \mu \mathrm{g} / \mathrm{kg}$, 
6 injections/d at hourly intervals, twice weekly for 6 wk (32). Control mice received the same number of injections of sterile saline vehicle. The cerulein preparation consisted of a stock solution of $1 \mathrm{mg}$ of cerulein dissolved in $1 \mathrm{~mL}$ of $50 \mathrm{mM} \mathrm{NaOH}$. The working solution consisted of $5 \mu \mathrm{L}$ of stock solution in $1 \mathrm{~mL}$ of sterile saline $(5 \mu \mathrm{g} / \mathrm{mL})$.

\section{Synthesis and Administration of ${ }^{125}$ I-lodo-DPA-713}

${ }^{125}$ I-iodo-DPA-713 was synthesized as described previously (33), and batches ranging from 70.3 to $77.7 \mathrm{GBq} / \mathrm{mmol}(1,900-2,100 \mathrm{Ci} /$ $\mathrm{mmol}$ ) in specific radioactivity were used. The ${ }^{125}$ I-iodo-DPA-713 radiotracer was formulated in $10 \%$ ethanol in phosphate-buffered saline (PBS) at $\mathrm{pH} 7.4$ and was injected as a $100-\mu \mathrm{L}$ bolus through the tail vein.

\section{SPECT/CT Imaging}

Three mice from each group (treated and control) were injected with an equal amount of ${ }^{125}$ I-iodo-DPA-713 (37 MBq, $\left.1 \mathrm{mCi}\right)$ and were scanned in pairs $24 \mathrm{~h}$ later using an X-SPECT SPECT/CT scanner (Gamma Medica Ideas). Beforehand, the mice were anesthetized using $3 \%$ isoflurane in oxygen $(2 \mathrm{~L} / \mathrm{min})$, and during scanning the anesthesia was maintained using $2 \%$ isoflurane in oxygen. CT and SPECT data were reconstructed and coregistered using the manufacturer's software, and the data were displayed and analyzed using AMIDE (http://amide.sourceforge.net).

\section{Biodistribution of 125 |-lodo-DPA-713}

Six mice from each group were injected intravenously with 259 $\mathrm{kBq}(7 \mu \mathrm{Ci})$ of ${ }^{125} \mathrm{I}$-iodo-DPA-713 and placed back in their cages for $24 \mathrm{~h}$. Then, the mice were sacrificed by cervical dislocation, 15 tissues of interest were removed, a blood sample was taken by cardiac puncture, the intestinal contents were removed, and all tissues were briefly rinsed with water and blotted dry. The tissues were then weighed and counted using an automated $\gamma$-counter (1282 Compugamma CS; LKB Nuclear) with decay correction along with 2 dilutions of a standard injected dose. All values are expressed as percentage injected dose (\%ID) per gram of tissue.

\section{${ }^{18}$ F-FDG PET Imaging}

Three mice from each group were kept fasting for $4 \mathrm{~h}$ before receiving an intravenous injection of approximately $9.25 \mathrm{MBq}(\sim 250 \mu \mathrm{Ci})$

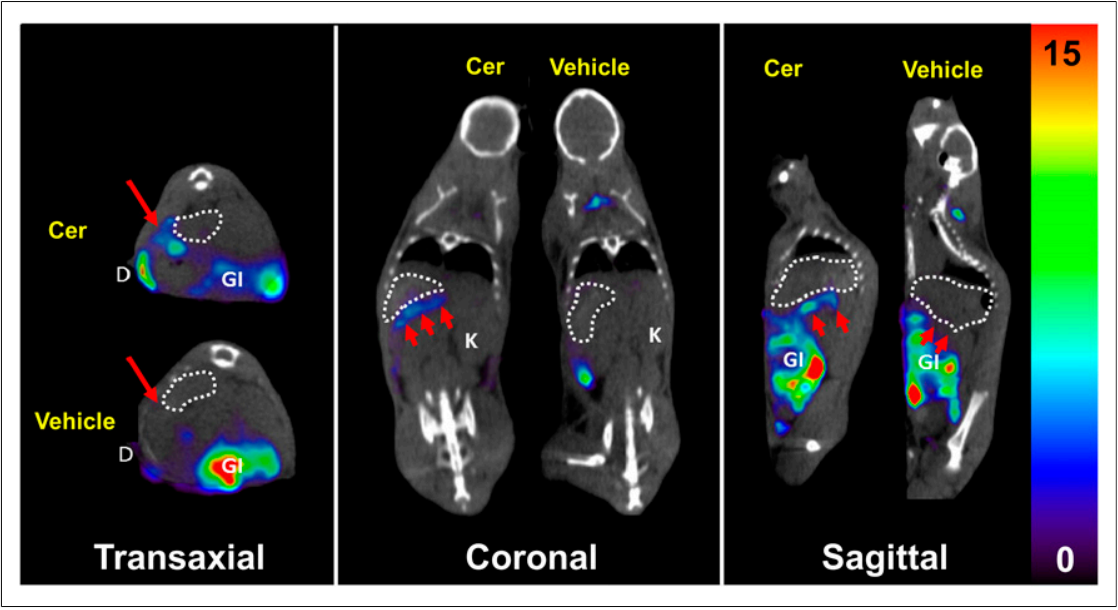

FIGURE 1. 125/-iodo-DPA-713 SPECT/CT of treated (Cer) and control (vehicle) mice. Coregistered images show radiotracer uptake in inflamed pancreas (arrows) and duodenum of treated mouse but not in either tissue of control mouse. Stomach is delineated by dotted circles. Gastrointestinal uptake of radiotracer is specific for peritoneal macrophages. Scale unit is \%ID/g. $\mathrm{D}=$ duodenum; $\mathrm{Gl}$ = gastrointestinal tract; $\mathrm{K}=$ kidney. of ${ }^{18}$ F-FDG (PET Net Solutions). After a $45-\mathrm{min}$ period of uptake with the mice under isoflurane anesthesia ( $2 \%$ isoflurane in oxygen at $2 \mathrm{~L} / \mathrm{min}$ ), they were scanned in cerulein-control pairs. The same anesthesia conditions were maintained during scanning. A SuperARGUS PET/CT scanner (Sedecal) was used, with a 20-min static acquisition per bed position. The PET and CT data were reconstructed using the manufacturer's software and were coregistered using AMIDE. Regions of interest, including pancreas and liver, were drawn, and ${ }^{18}$ F-FDG uptake was expressed as a ratio between treated and control mice.

\section{Near-Infrared Fluorescence Imaging}

DPA-713-IRDye800CW, a red-shifted fluorescent analog of DPA713-IRDye680LT $(30,31)$ (patent WO2013138612 A1), was formulated in $10 \%$ dimethylsulfoxide in PBS at $\mathrm{pH} 7.5$ and injected into one mouse from each group. After a 24-h period of uptake, the mice were sacrificed by cervical dislocation, the body cavity was exposed, and images were acquired using a Pearl Impulse Imager (LI-COR Biosciences) with a band-pass filter of $790 / 800 \mathrm{~nm}$ for the DPA-713IRDye $800 \mathrm{CW}$ probe. A white-light photograph was also taken. The images were displayed using the manufacturer's software (Image Studio, version 4.0).

\section{Epifluorescence Microscopy}

Tissues from the 2 mice injected with DPA-713-IRDye800CW were collected immediately after near-infrared fluorescence imaging, frozen over dry ice, cryosectioned into $20-\mu \mathrm{m}$ slices, and placed on charged glass slides. The sections were then probed for CD68 expression to delineate macrophages (34) within the tissue, as well as for expression of TSPO, the molecular target for DPA-713 analogs (35). The probing was done for $1 \mathrm{~h}$ at room temperature with a $\mathrm{pH} 7.5$ solution of PBS containing $10 \%$ fetal bovine serum (Gibco), mouse anti-CD68 antibody (IgG1, ab955, 1:67; Abcam), and rabbit polyclonal anti-TSPO antibody (NBP1-45769, 1:67; Novus Biologicals). Afterward, the slides were washed with PBS twice, for 5 min each time, and then probed with goat anti-mouse secondary antibody-fluorescein conjugate (ab97022, 1:250; Abcam) and chicken anti-rabbit Alexa Fluor 647 (A-21443, 1:250; Invitrogen) in PBS for $30 \mathrm{~min}$ at room temperature. After aspiration of the secondary antibody solution, the slides were exposed to Hoechst 33342 dye for 1.5 min (H3570, 1:1,000 in PBS; Invitrogen). The slides then underwent two more 5-min PBS washes, followed by the addition of aqueous mounting medium (Faramount; Dako) and a glass coverslip. The slides were then immediately viewed using an 80i upright epifluorescence microscope (Nikon) equipped with a DS-Qi1 monochrome dark-field chargecoupled-device camera (Nikon) and excited by a Intensilight C-HGFI lamp ( $\leq 720 \mathrm{~nm}$; Nikon) and an LB/LB-30 xenon lamp (790 nm; Sutter Instruments $\mathrm{Co}$.). Images were recorded and processed using NIS-Elements software (Nikon).

\section{Statistics}

$P$ values were calculated using a paired, 2-tailed $t$ test (Microsoft Excel), with 0.05 or less being considered significant.

\section{RESULTS}

\section{SPECT/CT Imaging}

In Figure 1 - the SPECT/CT images from 1 of the 3 pairs of mice-a thin line of ${ }^{125}$ I-iodo-DPA-713 uptake is seen adjacent 


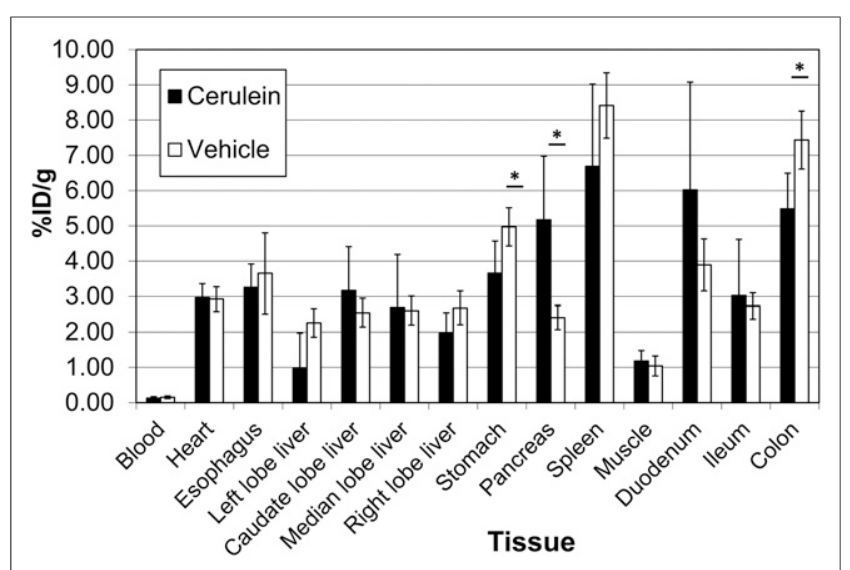

FIGURE 2. Ex vivo biodistribution in 4 treated (cerulein) and 4 control (vehicle) mice injected intravenously with $259 \mathrm{kBq}(7 \mu \mathrm{Ci})$ of ${ }^{125}$-iodoDPA-713. The indicated tissues were collected and weighed, and their radioactivity was counted along with a standard dose for comparison. Data are mean \pm SD. ${ }^{\star} P<0.05$

to and posterior to stomach in the treated mouse but not in the control mouse. The pattern shows an inflamed pancreas and a portion of duodenum in the treated mouse. Proximal duodenum in the treated mouse retained radiotracer, whereas duodenum in the control mouse did not. The other 2 pairs of mice showed similar differences in uptake in pancreas, spleen, and duodenum (Supplemental Fig. 1; supplemental materials are available at http://jnm.snmjournals.org), and all mice showed uptake in the lower gastrointestinal tract, possibly reflecting uptake by abundant sentinel peritoneal macrophages as well as hepatobiliary clearance of radiotracer $(36,37)$. Uptake of ${ }^{125}$ I-iodo-DPA-713 in gastrointestinal tract, brown fat, and kidneys was previously demonstrated to be specific binding via the observance of near-quantitative pharmacologic blockade with unlabeled iodo-DPA-713 (31).

\section{Biodistribution of}

125I-lodo-DPA-713

The ex vivo ${ }^{125}$ I-iodo-DPA-713 biodistribution (Fig. 2) was similar between groups for all assayed tissues except stomach

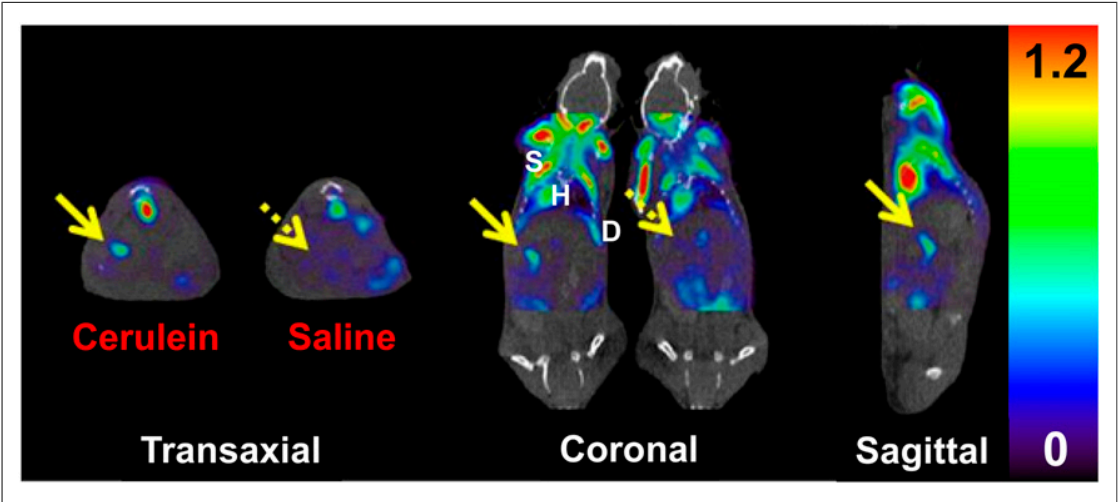

FIGURE 3. ${ }^{18} \mathrm{~F}-F D G$ PET/CT of treated (cerulein) and control (saline) mice. Coregistered images show radiotracer uptake in inflamed pancreas (solid arrow) of cerulein-treated mouse, with reduced background uptake (dashed arrow) seen in control mouse. Prominent uptake is also apparent in skeletal muscle, heart, and diaphragm. Scale unit is SUV (\%ID/g/body weight). $\mathrm{D}=$ diaphragm; $\mathrm{H}=$ heart; $\mathrm{S}=$ skeletal muscle. (mean $\pm \mathrm{SD}, 3.66 \pm 0.91$ vs. $4.97 \pm 0.54$ in cerulein vs. control, respectively, $P=0.04)$, pancreas $(5.17 \pm 1.18$ vs. $2.41 \pm 0.34, P=$ $0.02)$, duodenum ( $6.04 \pm 3.06$ vs. $3.89 \pm 0.74, P=0.26)$, and colon $(5.48 \pm 1.01$ vs. $7.43 \pm 0.82, P=0.01)$. Notably, only in pancreas was uptake higher for the treated animals than for the control animals. The larger SD in treated mice relative to control mice reflected the expected heterogeneity in phenotypic severity in this model (38-40) and accounted for the insignificance of the 1.5-fold average increase in duodenal uptake in treated over control mice.

\section{${ }^{18}$ F-FDG PET Imaging}

${ }^{18} \mathrm{~F}-\mathrm{FDG}$ uptake was high in heart, in muscle, and within the gastrointestinal tract in both groups of mice, with pancreas being evident in both groups as well (Fig. 3). Two of the treated mice displayed modestly increased radiotracer uptake in the pancreas, whereas the third had triple the uptake of the matched control mouse, again in keeping with the anticipated phenotypic variability of the model (38). Pancreas-to-liver ratios were measured from each set of PET SUV data, with the average being $2.86 \pm 1.05$ for treated pancreas and $1.57 \pm 0.55$ for control pancreas. The average cerulein-to-control pancreas-to-liver ratio was $1.96 \pm 0.86$. A positive finding for inflammation using this method is defined as pancreas uptake higher than background uptake, with liver or gastrointestinal tract serving as the comparative background tissue (41).

\section{Near-Infrared Fluorescence Imaging}

Figure 4, an image of DPA-713-IRDye800CW-injected treated and control mice side by side, reveals the presence of DPA-713IRDye $800 \mathrm{CW}$ conjugate in pancreas, in portions of liver, and focally within gastrointestinal tract in the treated mouse, as well as excretion through the urinary bladder in both animals. DPA713-IRDye800CW is displayed as a rainbow color scheme. Spleen and colon are not visible in this image. Only the treated animal retained DPA-713-IRDye800CW in upper gastrointestinal tissues and within pancreas and portions of liver. The control animal showed only urinary clearance of the conjugate, demonstrating inflammation within cerulein-affected tissues.

\section{Epifluorescence Microscopy}

Figure 5 shows fluorescence micrographs of pancreatic tissue depicting the ex vivo disposition of DPA-713-IRDye800CW in relation to CD68-expressing macrophages and total TSPO expression. TSPO is expressed in pancreas, a neuroendocrine tissue $(42,43)$. DPA-713 analogs target TSPO, but radioiodinated DPA-713 and DPA-713-IRDyes were previously demonstrated to be selectively trapped by activated macrophages after a 24-h biologic uptake period (44). The micrographs in Figure 5 demonstrate that DPA-713-IRDye800CW was trapped within CD68-expressing macrophages, which were abundant within treated pancreas. Equally abundant endogenous TSPO expression was present throughout both treated and control pancreas but was not associated with DPA-713-IRDye800CW retention after the 24-h uptake period. DPA-713-IRDye800CW was retained in small amounts within the CD68-expressing macrophages of control pancreas (Supplemental Fig. 2). The data demonstrate that DPA-713-IRDye $800 \mathrm{CW}$ - and, by 


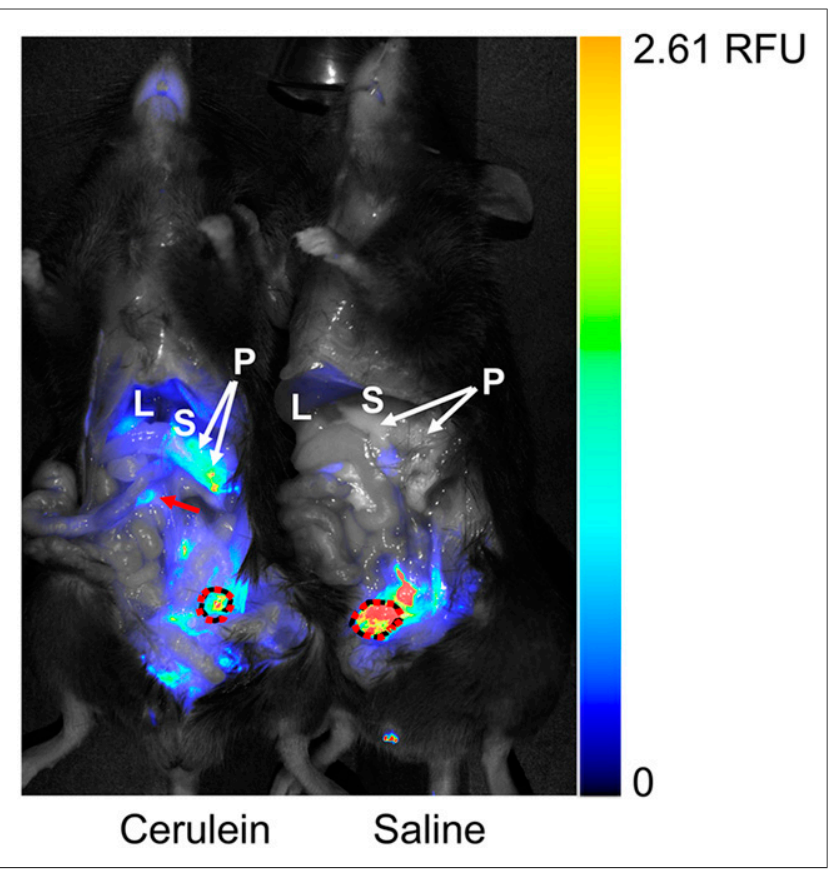

FIGURE 4. EX vivo near-infrared fluorescence imaging of DPA-713IRDye $800 \mathrm{CW}$ in treated (cerulein) and control (saline) mice. Fluorescent tracer uptake is seen in pancreas, gastrointestinal tract (arrow), and urinary bladder (dotted circle) of treated mouse but in only urinary bladder of control mouse. Adjacent stomach is labeled for context. Color bar is relative fluorescence units (RFU). $L=$ liver; $P=$ pancreas; $S=$ stomach.

shared structural analogy, ${ }^{125}$ I-iodo-DPA-713, which has the same targeting mechanism-is selectively trapped within and report on activated macrophages within pancreas after a 24-h uptake period. Indeed, the radiotracer and fluorescent tracer uptake patterns displayed in Figures 1 and 4, respectively, share the same distribution.

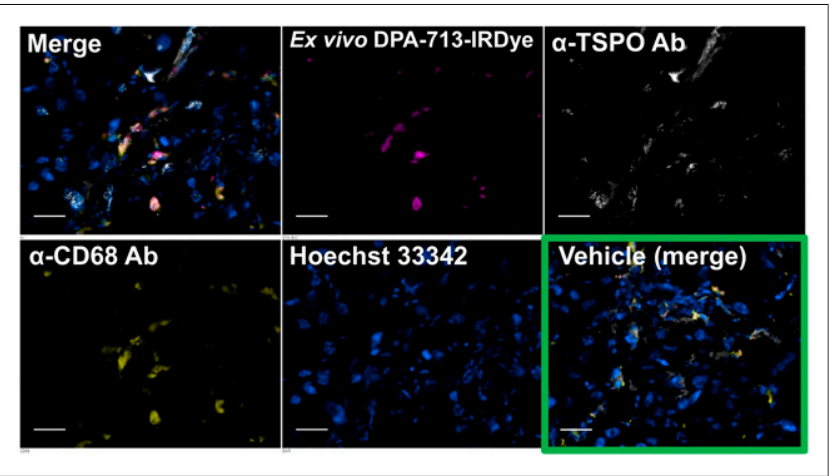

FIGURE 5. Ex vivo near-infrared fluorescence microscopy of DPA-713-IRDye800CW in pancreatic sections from mice in Figure 4. Separate and merged images are shown for treated mouse, whereas only merged image is shown for control mouse (vehicle). Treated pancreas has abundant fluorescent tracer uptake (magenta) in CD68-positive phagocytic cells (yellow) along with abundant TSPO expression (white), whereas control pancreas has almost no phagocytic cells present, small amounts of TSPO expression, and no visible fluorescent probe uptake. Scale bar $=50 \mu \mathrm{m} . \mathrm{Ab}=$ antibody.

\section{DISCUSSION}

Here, we have described the visualization and quantification of macrophage-specific inflammation using both ${ }^{125}$ I-iodoDPA-713 SPECT/CT and DPA-713-IRDye800CW near-infrared fluorescence imaging within the cerulein mouse model of chronic pancreatitis. ${ }^{18}$ F-FDG PET was also performed to compare metabolic imaging of inflammation with the cell-specific imaging. ${ }^{125} \mathrm{I}-$ iodo-DPA-713 SPECT/CT revealed starkly differential radiotracer retention in cerulein-treated pancreas (Fig. 1 and Supplemental Fig. 1) and, in some mice, duodenum, whereas control mice exhibited no radiotracer retention in these tissues. The biodistribution of ${ }^{125}$ I-iodo-DPA-713 (Fig. 2) revealed significant differences in radiotracer uptake between the treated and control groups in pancreas and, oppositely, in stomach, where other differences in uptake were insignificant. Qualitative imaging of macrophages in gastrointestinal tissues using DPA-713IRDye $800 \mathrm{CW}$ near-infrared fluorescence revealed uptake of the fluorescent probe in pancreas, duodenum, and portions of liver (Fig. 4) in the treated mouse, whereas the control mouse showed the fluorescent probe only in the urinary bladder. Collection of the pancreas from both mice, cryosectioning, and costaining with anti-CD68 and anti-TSPO antibodies revealed that accumulation of DPA-713-IRDye $800 \mathrm{CW}$ was confined to CD68-expressing macrophages after a 24 -h in vivo uptake period despite the presence of widespread TSPO expression within the pancreas (Fig. 5 and Supplemental Fig. 2). Lastly, in the 3 pairs of mice that underwent ${ }^{18} \mathrm{~F}-\mathrm{FDG}$ PET/CT to compare metabolic imaging with macrophage-specific imaging, ${ }^{18} \mathrm{~F}$-FDG uptake in pancreata was present in only 2 treated mice but, though reduced by $55 \%$, was present in all 3 control mice (Supplemental Fig, 3). In this study, ${ }^{125}$ I-iodo-DPA713 imaging yielded an unambiguous high-contrast qualitative read on pancreatic and upper gastrointestinal inflammation whereas ${ }^{18} \mathrm{~F}$-FDG imaging required quantitation and comparison with a reference tissue (liver) to accurately assess inflammatory status.

To our knowledge, no molecular probe other than ${ }^{18} \mathrm{~F}$-FDG PET has been reported for imaging chronic pancreatitis, and although ${ }^{18} \mathrm{~F}-\mathrm{FDG}$ PET is the emerging clinical standard for imaging pancreatic inflammation (41), ${ }^{18} \mathrm{~F}$-FDG is taken up by all cells actively using glucose (45-48), including cells in both normal pancreas and pancreatic tumors $(15,16,49)$.

Amid the dense mixed-lineage inflammatory recruitment to sites of disease, ${ }^{125}$ I-iodo-DPA-713 is able to single out and provide a measurement of macrophage content for pure readout of phagocyte-specific inflammation. That narrow target population could serve as a tool to detect subtle chronic pancreatitis but could also be useful in acute pancreatitis to differentiate mild, organconfined disease from lethal, systemic disease, occurring in 3\%$16 \%$ of patients (50). Systemic inflammatory disease of pancreatic origin in critical tissues such as lung, liver, and peritoneum is characterized by activation of resident macrophages into proinflammatory M1 phenotype cells $(51,52)$. These can be visualized and localized by ${ }^{125}$ I-iodo-DPA-713 imaging, which detects all mature phagocytes.

Imaging with radiolabeled analogs of ${ }^{125}$ I-iodo-DPA-713, including ${ }^{123 / 124}$ I-iodo-DPA-713, does not require fasting and is specific for macrophages $24 \mathrm{~h}$ after injection, potentially distinguishing inflammation from pancreatic neoplasms. Low and uniform background liver uptake also allows for higher-contrast 
pancreatic radiotracer uptake as well as visualization of any downstream duodenal or peritoneal inflammatory uptake. These cell-specific and favorable nontarget clearance features of iodoDPA-713 make it a promising radiotracer for imaging pancreatic and biliary inflammation.

\section{CONCLUSION}

Iodo-DPA-713 imaging represents a view of macrophage-specific inflammation in the cerulein model of mild chronic pancreatitis. Because of its cell-type specificity and clearance from nontarget tissues, radiolabeled iodo-DPA-713 provides a high-contrast view of macrophage infiltration within the pancreas and surrounding tissues, including liver and duodenum, delineating inflammationspecific disease in the upper gastrointestinal tract and nearby regions. Because of its physical half-life of $4 \mathrm{~d}$, use of ${ }^{124}$ I-iodoDPA-713 PET may enable clinical translation of this method by allowing for the 24-h uptake period, as well as for shipping to sites distant from the production site.

\section{DISCLOSURE}

This work was supported by grants to Martin Pomper from the National Institutes of Health (EB009367) and the Stabler Foundation and to Pankaj Pasricha from the National Institute of Diabetes and Digestive and Kidney Diseases (DK 073558). No other potential conflict of interest relevant to this article was reported.

\section{ACKNOWLEDGMENT}

We thank Gilbert Green for operating the small-animal SPECT/ CT scanner.

\section{REFERENCES}

1. Yadav D, Lowenfels AB. The epidemiology of pancreatitis and pancreatic cancer. Gastroenterology. 2013;144:1252-1261.

2. Tinto A, Lloyd DA, Kang JY, et al. Acute and chronic pancreatitis: diseases on the rise-a study of hospital admissions in England 1989/90-1999/2000. Aliment Pharmacol Ther. 2002;16:2097-2105.

3. Jupp J, Fine D, Johnson CD. The epidemiology and socioeconomic impact of chronic pancreatitis. Best Pract Res Clin Gastroenterol. 2010;24:219-231.

4. Peery AF, Crockett SD, Barritt AS, et al. Burden of gastrointestinal, liver, and pancreatic diseases in the United States. Gastroenterology. 2015;149: 1731-1741.

5. Majumder S, Chari ST. Chronic pancreatitis. Lancet. 2016;387:1957-1966.

6. Xu S, Chheda C, Ouhaddi Y, et al. Characterization of mouse models of early pancreatic lesions induced by alcohol and chronic pancreatitis. Pancreas. 2015; 44:882-887.

7. Saeki K, Kanai T, Nakano M, et al. CCL2-induced migration and SOCS3mediated activation of macrophages are involved in cerulein-induced pancreatitis in mice. Gastroenterology. 2012;142:1010-1020.

8. Detlefsen S, Sipos B, Zhao J, Drewes AM, Kloppel G. Autoimmune pancreatitis: expression and cellular source of profibrotic cytokines and their receptors. Am J Surg Pathol. 2008;32:986-995.

9. Hietaranta A, Mustonen H, Puolakkainen P, Haapiainen R, Kemppainen E. Proinflammatory effects of pancreatic elastase are mediated through TLR4 and NF-кB. Biochem Biophys Res Commun. 2004;323:192-196.

10. Bang JY, Varadarajulu S. Neoplasia in chronic pancreatitis: how to maximize the yield of endoscopic ultrasound-guided fine needle aspiration. Clin Endosc. 2014;47: 420-424.

11. Berger LA, Rhodes JM, Agnew JE, et al. Screening for pancreatic disease: a comparison of grey-scale ultrasonography and isotope scanning. Lancet. 1979;1: 633-635.

12. Gumaste VV. Diagnostic tests for acute pancreatitis. Gastroenterologist. 1994; 2:119-130.
13. Nesvaderani M, Eslick GD, Cox MR. Acute pancreatitis: update on management. Med J Aust. 2015;202:420-423.

14. Van Dyke JA, Stanley RJ, Berland LL. Pancreatic imaging. Ann Intern Med. 1985;102:212-217.

15. Pery C, Meurette G, Ansquer C, Frampas E, Regenet N. Role and limitations of ${ }^{18}$ F-FDG positron emission tomography (PET) in the management of patients with pancreatic lesions. Gastroenterol Clin Biol. 2010;34:465-474.

16. Strauss LG. Fluorine-18 deoxyglucose and false-positive results: a major problem in the diagnostics of oncological patients. Eur J Nucl Med. 1996;23:1409-1415.

17. Emmrich J, Weber I, Nausch M, et al. Immunohistochemical characterization of the pancreatic cellular infiltrate in normal pancreas, chronic pancreatitis and pancreatic carcinoma. Digestion. 1998;59:192-198.

18. Batarseh A, Papadopoulos V. Regulation of translocator protein $18 \mathrm{kDa}$ (TSPO) expression in health and disease states. Mol Cell Endocrinol. 2010;327: $1-12$.

19. Ching AS, Kuhnast B, Damont A, Roeda D, Tavitian B, Dolle F. Current paradigm of the $18-\mathrm{kDa}$ translocator protein (TSPO) as a molecular target for PET imaging in neuroinflammation and neurodegenerative diseases. Insights Imaging. 2012;3:111-119.

20. Selvaraj V, Stocco DM. The changing landscape in translocator protein (TSPO) function. Trends Endocrinol Metab. 2015;26:341-348.

21. Venneti S, Lopresti BJ, Wiley CA. Molecular imaging of microglia/macrophages in the brain. Glia. 2013;61:10-23.

22. Abourbeh G, Theze B, Maroy R, et al. Imaging microglial/macrophage activation in spinal cords of experimental autoimmune encephalomyelitis rats by positron emission tomography using the mitochondrial $18 \mathrm{kDa}$ translocator protein radioligand $\left[{ }^{18}\right.$ F]DPA-714. J Neurosci. 2012;32:5728-5736.

23. Awde AR, Boisgard R, Theze $\mathrm{B}$, et al. The translocator protein radioligand ${ }^{18} \mathrm{~F}$ DPA-714 monitors antitumor effect of erufosine in a rat 9L intracranial glioma model. J Nucl Med. 2013;54:2125-2131.

24. Buck JR, McKinley ET, Hight MR, et al. Quantitative, preclinical PET of translocator protein expression in glioma using ${ }^{18} \mathrm{~F}-\mathrm{N}$-fluoroacetyl- $\mathrm{N}$-(2,5-dimethoxybenzyl)-2-phenoxyaniline. J Nucl Med. 2011;52:107-114.

25. Cerami C, Perani D. Imaging neuroinflammation in ischemic stroke and in the atherosclerotic vascular disease. Curr Vasc Pharmacol. 2015;13:218-222.

26. Chen Y, Sajjad M, Wang Y, Batt C, Nabi HA, Pandey RK. TSPO 18 kDa (PBR) targeted photosensitizers for cancer imaging (PET) and PDT. ACS Med Chem Lett. 2010;2:136-141.

27. Corcia P, Tauber C, Vercoullie J, et al. Molecular imaging of microglial activation in amyotrophic lateral sclerosis. PLoS One. 2012;7:e52941.

28. Coughlin JM, Wang Y, Munro CA, et al. Neuroinflammation and brain atrophy in former NFL players: an in vivo multimodal imaging pilot study. Neurobiol Dis. 2015;74:58-65.

29. Doorduin J, Klein HC, Dierckx RA, James M, Kassiou M, de Vries EF. $\left[{ }^{11} \mathrm{C}\right]-$ DPA-713 and $\left[{ }^{18} \mathrm{~F}\right]$-DPA-714 as new PET tracers for TSPO: a comparison with $\left[{ }^{11} \mathrm{C}\right]-(\mathrm{R})-\mathrm{PK} 11195$ in a rat model of herpes encephalitis. Mol Imaging Biol. 2009;11:386-398.

30. Foss CA, Bedja D, Mease RC, et al. Molecular imaging of inflammation in the ApoE-1- mouse model of atherosclerosis with iodoDPA. Biochem Biophys Res Commun. 2015;461:70-75.

31. Foss CA, Harper JS, Wang H, Pomper MG, Jain SK. Noninvasive molecular imaging of tuberculosis-associated inflammation with radioiodinated DPA-713. J Infect Dis. 2013;208:2067-2074.

32. Aghdassi AA, Mayerle J, Christochowitz S, Weiss FU, Sendler M, Lerch MM. Animal models for investigating chronic pancreatitis. Fibrogenesis Tissue Repair. 2011;4:26.

33. Wang H, Pullambhatla M, Guilarte TR, Mease RC, Pomper MG. Synthesis of [ ${ }^{125}$ I] iodoDPA-713: a new probe for imaging inflammation. Biochem Biophys Res Commun. 2009;389:80-83.

34. da Silva RP, Platt N, de Villiers JS, Gordon S. Membrane molecules and macrophage endocytosis: scavenger receptor and macrosialin as markers of plasma-membrane and vacuolar functions. Biochem Soc Trans. 1996;24: 220-224.

35. Damont A, Roeda D, Dolle F. The potential of carbon- 11 and fluorine- 18 chemistry: illustration through the development of positron emission tomography radioligands targeting the translocator protein $18 \mathrm{kDa}$. J Labelled Comp Radiopharm. 2013;56:96-104.

36. Kurushima H, Ramprasad M, Kondratenko N, Foster DM, Quehenberger O, Steinberg D. Surface expression and rapid internalization of macrosialin (mouse CD68) on elicited mouse peritoneal macrophages. J Leukoc Biol. 2000;67:104-108.

37. Sakai Y, Masamune A, Satoh A, Nishihira J, Yamagiwa T, Shimosegawa T. Macrophage migration inhibitory factor is a critical mediator of severe acute pancreatitis. Gastroenterology. 2003;124:725-736. 
38. Su KH, Cuthbertson C, Christophi C. Review of experimental animal models of acute pancreatitis. HPB (Oxford). 2006;8:264-286.

39. van Westerloo DJ, Florquin S, de Boer AM, et al. Therapeutic effects of troglitazone in experimental chronic pancreatitis in mice. Am J Pathol. 2005;166:721728.

40. Zion O, Genin O, Kawada N, et al. Inhibition of transforming growth factor beta signaling by halofuginone as a modality for pancreas fibrosis prevention. Pancreas. 2009;38:427-435.

41. Santhosh S, Mittal BR, Rana SS, et al. Metabolic signatures of malignant and non-malignant mass-forming lesions in the periampulla and pancreas in FDG PET/CT scan: an atlas with pathologic correlation. Abdom Imaging. 2015;40:1285-1315.

42. Brody AL, Okita K, Shieh J, et al. Radiation dosimetry and biodistribution of the translocator protein radiotracer $\left[{ }^{11} \mathrm{C}\right] \mathrm{DAA} 1106$ determined with $\mathrm{PET} / \mathrm{CT}$ in healthy human volunteers. Nucl Med Biol. 2014;41:871-875.

43. Endres CJ, Coughlin JM, Gage KL, Watkins CC, Kassiou M, Pomper MG. Radiation dosimetry and biodistribution of the TSPO ligand ${ }^{11} \mathrm{C}-\mathrm{DPA}-713$ in humans. J Nucl Med. 2012;53:330-335.

44. Ordonez AA, Pokkali S, DeMarco VP, et al. Radioiodinated DPA-713 imaging correlates with bactericidal activity of tuberculosis treatments in mice. Antimicrob Agents Chemother. 2015;59:642-649.
45. Mankoff DA, Dehdashti F, Shields AF. Characterizing tumors using metabolic imaging: PET imaging of cellular proliferation and steroid receptors. Neoplasia. 2000;2:71-88.

46. Smith TA. Mammalian hexokinases and their abnormal expression in cancer. Br J Biomed Sci. 2000;57:170-178.

47. Strauss LG. Sensitivity and specificity of positron emission tomography (PET) for the diagnosis of lymph node metastases. Recent Results Cancer Res. 2000;157:12-19.

48. Phelps ME. PET: the merging of biology and imaging into molecular imaging. J Nucl Med. 2000;41:661-681.

49. Rijkers AP, Valkema R, Duivenvoorden HJ, van Eijck CH. Usefulness of F-18fluorodeoxyglucose positron emission tomography to confirm suspected pancreatic cancer: a meta-analysis. Eur J Surg Oncol. 2014;40:794-804.

50. Fu CY, Yeh CN, Hsu JT, Jan YY, Hwang TL. Timing of mortality in severe acute pancreatitis: experience from 643 patients. World J Gastroenterol. 2007;13: 1966-1969.

51. Gea-Sorlí S, Closa D. Role of macrophages in the progression of acute pancreatitis. World J Gastrointest Pharmacol Ther. 2010;1:107-111.

52. Shrivastava P, Bhatia M. Essential role of monocytes and macrophages in the progression of acute pancreatitis. World J Gastroenterol. 2010;16:39954002 . 